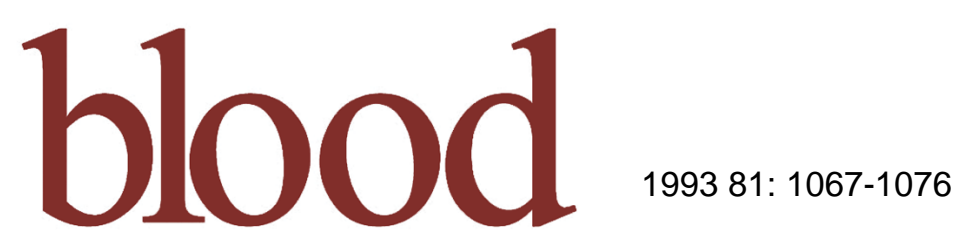

\title{
Quantitative assessment of erythropoiesis and functional classification of anemia based on measurements of serum transferrin receptor and erythropoietin
}

Y Beguin, GK Clemons, P Pootrakul and G Fillet

Information about reproducing this article in parts or in its entirety may be found online at: http://bloodjournal.hematologylibrary.org/misc/rights.dtl\#repub_requests

Information about ordering reprints may be found online at:

http://bloodjournal.hematologylibrary.org/misc/rights.dtl\#reprints

Information about subscriptions and ASH membership may be found online at:

http://bloodjournal.hematologylibrary.org/subscriptions/index.dtl

Blood (print ISSN 0006-4971, online ISSN 1528-0020), is published semimonthly by the American Society of Hematology, 1900 M St, NW, Suite 200, Washington DC 20036.

Copyright 2007 by The American Society of Hematology; all rights reserved.

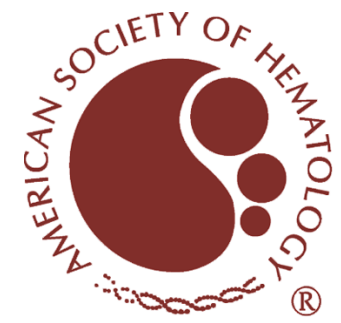




\title{
Quantitative Assessment of Erythropoiesis and Functional Classification of Anemia Based on Measurements of Serum Transferrin Receptor and Erythropoietin
}

\author{
By Yves Beguin, Gisela K. Clemons, Pensri Pootrakul, and Georges Fillet
}

\begin{abstract}
We evaluated the quantitative value of a simple model of erythropoiesis, based on the basic assumptions that the red blood cell (RBC) mass determines erythropoietin (Epo) production, which in turn stimulates erythropoietic activity. The RBC mass was quantitated by direct isotopic measurement (RCM), Epo production by serum Epo levels, and erythropoiesis by the ferrokinetic measurement of the erythron transferrin uptake (ETU), the serum transferrin receptor (TfR) level, and the reticulocyte (retic) index, and was completed by an evaluation of overall marrow erythron cellularity. We studied a total of 195 subjects, including 31 normal individuals, 38 patients with polycythemia, and 126 patients with various forms of anemia. Instead of only quantitating Epo and erythropoiesis in absolute terms, we also evaluated them in relation to the degree of anemia or polycythemia, and expressed the results as a ratio of observed values to values predicted from the regression equations between hematocrit (Hct) on the one hand, and Epo, TfR, and ETU on the other, obtained in a carefully selected subpopulation. The slope of the regression of TfR (as well as ETU) versus Hct was very similar to the slope of the regression of Epo versus Hct. Average EPO and TfR (as well as ETU) values predicted from the regression equations were quite comparable to observed values in most
\end{abstract}

$\mathbf{E}^{\mathrm{R}}$ RYTHROPOIESIS depends on the proliferative capacity of erythroid progenitors in the bone marrow (BM) and their stimulation by erythropoietin (Epo). Epo production by the kidney is determined by the level of oxygen supply, which depends mostly on the red blood cell (RBC) mass. ${ }^{1}$ An inverse relationship has been shown between serum Epo levels and hematocrit $(\mathrm{Hct}){ }^{2}$ Epo stimulates $\mathrm{RBC}$ production by inducing the proliferation and differentiation of committed erythroid progenitors. In the presence of a normal marrow stem cell reserve, erythropoiesis will therefore increase in proportion to the degree of anemia. On the other hand, polycythemia can originate from an autonomous proliferation of marrow erythroid progenitors (polycythemia vera) or be secondary to increased Epo production.

The present study is based on a simple model of the regulation of erythropoiesis, ie, that the RBC mass determines Epo production, which in turn stimulates erythropoietic activity. Erythropoiesis was quantitated both by the ferrokinetic measurement of the erythron transferrin uptake (ETU) ${ }^{3}$ and the determination of plasma transferrin receptor (TfR) levels, which we recently showed to be a simple quantitative assay of total erythropoiesis in rats ${ }^{4}$ as well as in humans. ${ }^{5}$ This report is aimed at: (1) quantitating erythropoiesis relative to the Hct rather than simply in absolute values; (2) testing the validity of the model for the quantitative assessment of the erythropoietic response to Epo; (3) testing the appropriateness of a physiologic assessment of RBC disorders based on measurements of the Hct, retic index, serum Epo, and serum TfR in a single blood sample; and (4) examining the association between the pathogenesis of disordered erythropoiesis and clinical diagnosis. groups of subjects, with exceptions predictable from knowledge of the pathophysiology of these hematologic disorders. We identified four major patterns of erythropoiesis, ie, normal, hyperdestruction (with variants of hemolysis or ineffective erythropoiesis), intrinsic marrow hypoproliferation, and defective Epo production. Dissecting out groups of patients showed much greater heterogeneity than when patients were analyzed by group. This was particularly true in the case of a hypoproliferative component being combined with hyperdestruction, giving what we called a "mixed disorder of erythropoiesis." We conclude that the pathophysiology of anemia can be assessed by a simple measurement of Hct, retic index, Epo, and TfR levels, with Epo and TfR being more informative when expressed in relation to the degree of anemia. The model is particularly useful for detecting the presence of multiple mechanisms of anemia in the same patient. However, it has limitations inherent to the relative invalidity of $\mathrm{TfR}$ in iron deficiency, the imprecision of a retic count, and the difficulty in distinguishing hemolysis from ineffective erythropoiesis in some patients and in recognizing a component of hyperdestruction in hypoproliferative anemia.

(C) 1993 by The American Society of Hematology.

\section{SUBJECTS AND METHODS}

\section{Subjects}

A total of 195 subjects were studied. These included 31 normal subjects aged 18 to 76 years, 38 patients with polycythemia (PC), and 126 patients with anemia. The clinical diagnosis of $P C$ was based on the isotopic measurement of an RBC mass (RCM) greater than $125 \%$ of predicted value, ${ }^{6}$ either currently or previously. Among polycythemic patients, 9 had polycythemia vera (PV), 12 secondary PC, 12 PC of uncertain origin, and 5 relative $\mathrm{PC}$ (no history of absolute PC and current RCM lower than $125 \%$ of predicted value in the presence of a reduced plasma volume).$^{6,7}$ Among anemic patients, 18 had a myeloproliferative disorder (MPD) (either chronic myelogenous leukemia [CML] or essential thrombocythemia), 10 a myelodysplastic syndrome (MDS), 18 agnogeneic myeloid metaplasia

From the Department of Hematology, University of Liège, Liège, Belgium; the Lawrence Berkeley Laboratory, Division of Cellular and Molecular Biology, Berkeley, CA; and the Thalassemia Center, Faculty of Graduate Studies and Division of Hematology, Department of Medicine, Siriraj Hospital, Mahidol University, Bangkok, Thailand.

Submitted April 19, 1991; accepted October 14, 1992.

Supported in part by Grants No. HL22469 (G.K.C.) and HL34408 (P.P.) from the National Institutes of Health, by Grant No. 3.4555.9I (Y.B. and G.F.) from the Fund for Medical Scientific Research (FRSM, Belgium), and by a grant (Y.B. and G.F.) from the University of Liège School of Medicine.

Address reprint requests to Yves Beguin, MD, University of Liège, Department of Hematology, CHU Sart-Tilman, 4000 Liege, Belgium.

The publication costs of this article were defrayed in part by page charge payment. This article must therefore be hereby marked "advertisement" in accordance with 18 U.S.C. section 1734 solely to indicate this fact.

(C) 1993 by The American Society of Hematology.

0006-4971/93/8104-0020\$3.00/0 
(AMM), 11 hypoplastic/aplastic anemia, 15 chronic renal failure, 11 $\beta$-thalassemia/hemoglobin E, 10 hemoglobin $\mathrm{H}(\mathrm{HbH})$ disease, 8 hereditary spherocytosis, and 11 autoimmune hemolytic anemia (AIHA). In addition, 5 patients with chronic renal failure were receiving recombinant human erythropoietin $(40 \mathrm{U} / \mathrm{Kg}, 3$ times a week, intravenously [IV] after hemodialysis) and 9 patients had received an autologous $(\mathrm{N}=4)$ or allogeneic $(\mathrm{N}=5) \mathrm{BM}$ transplant (BMT) 6 to 12 months earlier. Informed consent was obtained from all subjects. The vast majority of them underwent the procedure as part of the routine investigation of their hematologic disorder. Except for marrow transplant patients, they were generally in steady state and had not received blood transfusions in the preceding 3 months. None of the patients was iron deficient, based on a serum ferritin level higher than $12 \mathrm{ng} / \mathrm{mL}^{8}$

\section{Blood Volume (BV) Measurement}

Total BV was the sum of simultaneous measurements of RCM and plasma volume. The patient's own plasma or a hepatitis-free and acquired immunodeficiency syndrome (AIDS)-free plasma from a normal donor was used, depending on the patient's unbound ironbinding capacity (UIBC). Ten milliliters of plasma was incubated for 30 minutes at $37^{\circ} \mathrm{C}$ with 2 to $3 \mu \mathrm{Ci}$ of ${ }^{59} \mathrm{FeCl}_{3}$ previously mixed with $4 \%$ sodium citrate to ensure a molar ratio of citrate to iron of $50: 1$. RBCs from $20 \mathrm{~mL}$ autologous whole blood were incubated for 30 minutes at $37^{\circ} \mathrm{C}$ with 10 to $20 \mu \mathrm{Ci}$ of $\mathrm{Na}_{2}{ }^{51} \mathrm{CrO}_{4}$, washed twice, and resuspended in normal saline. ${ }^{9}$ Weighed volumes of the labeled solutions were set aside for the preparation of diluted standard solutions. Weighed amounts of labeled RBCs and plasma were injected into a forearm vein and samples were drawn from the other arm. Plasma iron (PI) and total iron-binding capacity (TIBC) were measured by standard procedures. ${ }^{10,11}{ }^{59} \mathrm{Fe}$ and ${ }^{51} \mathrm{Cr}$ activities were counted in a gamma counter and corrections were made for cross-counting using standard solutions. ${ }^{55} \mathrm{Fe}$ was occasionally used in place of ${ }^{59} \mathrm{Fe}$ and its activity was counted in a liquid scintillation counter. Plasma volume and RCM were calculated as:

$$
\text { Plasma Volume or RCM }(\mathrm{mL})=\frac{I(\mathrm{cpm})}{\mathrm{C}(\mathrm{cpm} / \mathrm{mL})}
$$

where $I$ is the total amount of radioactivity injected, and $C$ is the sample activity per milliliter of plasma extrapolated to 0 time or per milliliter of RBCs in the 15- to 30-minute sample (60-minute sample when delayed mixing was suspected). The observed/predicted $(\mathrm{O} / \mathrm{P})$ ratio of RCM was calculated. ${ }^{6}$

\section{Ferrokinetic Studies}

The plasma iron turnover (PIT) was calculated from a standard formula ${ }^{12}$ :

PIT (mg/dL whole blood per day)

$$
=\frac{\operatorname{PI}(\mu \mathrm{g} / \mathrm{dL}) \times(100-\mathrm{Hct} \times 0.9) / 100}{\mathrm{t}-1 / 2(\mathrm{~min})}
$$

where $\mathrm{t}-1 / 2$ is the clearance half-time of transferrin- ${ }^{59} \mathrm{Fe}$, and 0.9 the correction for trapped plasma in the Hct $(0.98)$ and to convert venous Hct to whole body Het (0.92).

Extravascular flux, a function of plasma iron concentration, ${ }^{12}$ was then subtracted to obtain tissue iron uptake (TIU):

TIU (mg/dL whole blood per day)

$$
=\mathrm{PIT}-(\mathrm{PI} \times[100-\mathrm{Hct} \times 0.9] / 100 \times 0.0015)
$$

The effect of transferrin saturation (S) was removed by converting TIU to iron-bearing transferrin uptake, and nonerythron uptake was subtracted ${ }^{13}$ to obtain ETU:
ETU ( $\mu \mathrm{mol} / \mathrm{L}$ whole blood per day)

$$
=\frac{\mathrm{TIU} \times 10}{56} \times \frac{(200+2.2 \mathrm{~S})}{(200+6.4 \mathrm{~S})}-11
$$

The derivation of this formula is described elsewhere. ${ }^{3}$ ETU was also corrected for abnormalities in blood volume by the ratio of measured to predicted blood volume.

\section{Epo Assay}

Circulating Epo levels were measured by two different radioimmunoassays (RIA). The first RIA is a modification of an assay previously described in detail, ${ }^{2}$ and the second RIA is commercially available (Incstar Corp, Stillwater, MN). Both use recombinant human Epo (rHuEpo) for tracer and standards. Samples are incubated with rabbit anti-Epo serum for 3 days at $4^{\circ} \mathrm{C}$ (RIA \#1) or 2 hours at room temperature (RIA \#2) before Epo tracer is added. After overnight incubation, goat antirabbit serum is added. After centrifugation, the unbound tracer is removed by decantation and the pellet is counted. Several samples had to be diluted 1:10. Thirty samples, with Epo concentrations ranging from 10 to $200 \mathrm{mU} / \mathrm{mL}$, were measured by the two RIAs and no significant difference was found. Twelve control samples were run in each assay, with a between-assay coefficient of variation ranging from $10.3 \%$ to $14.1 \%$.

\section{$T f R$ Assay}

Human placental receptor-transferrin complex was purified as described elsewhere ${ }^{14}$ and injected repeatedly into rabbits. Serum IgG was isolated from rabbit serum ${ }^{15}$ and transferrin antibodies were removed by passing through a column of human diferric transferrin coupled to Affigel 15 (BioRad, Richmond, CA). Characterization of plasma TfR and receptor antibody has been described elsewhere. ${ }^{4,5}$

An enzyme-linked immunosorbent assay (ELISA) ${ }^{5}$ with minor modifications to measure serum levels of $T f R$ was used. Immunoplates I with certificate of quality (Nunc Intermed, Roskilde, Denmark) were used. The aliquots of blanks, standards, and unknown samples were added using a Digiflex automatic pipetor (Micromedic System, Philadelphia, PA). Standards were diluted to between 5 and $100 \mathrm{ng} /$ $\mathrm{mL}$ and unknown sera were diluted $1: 50$ to $1: 2,000$ with $0.15 \mathrm{~mol} /$ L phosphate-buffered saline (PBS; pH 7.4) containing $0.5 \%$ bovine serum albumin and $0.05 \%$ Tween 20 . After color development, differential absorbance was read in dual wave length mode at 492 and 690 in a Titertek Multiskan MCC/340 plate reader (Flow Laboratories, Herts, UK). In drawing the calibration curve, allowance was made for the molecular weight of the transferrin molecule included in the receptor-transferrin complex by multiplying the protein content of the standard by 0.69 . Each sample was run in triplicate. The between-assay variability (coefficient of variation) was $7.2 \%$ when the same control sample was measured in each plate.

\section{Reticulocyte (retic) Index}

Blood smears were stained with new methylene blue and reticulocytes in 1,000 cells were counted. The retic index was calculated after appropriate corrections for the subject's Hct and for reticulocyte shift, as described elsewhere. ${ }^{16}$ In the presence of anemia, a retic index greater than 3 is indicative of hemolytic anemia, and a retic index less than 2 of either a hypoproliferative or maturation abnormality.

\section{Statistical Methods}

Log transformed Epo, TfR, and ETU values were used in statistical analyses. Student's $t$-tests, with pooled or separated variances as appropriate, were used to compare two groups. Analysis of variance 


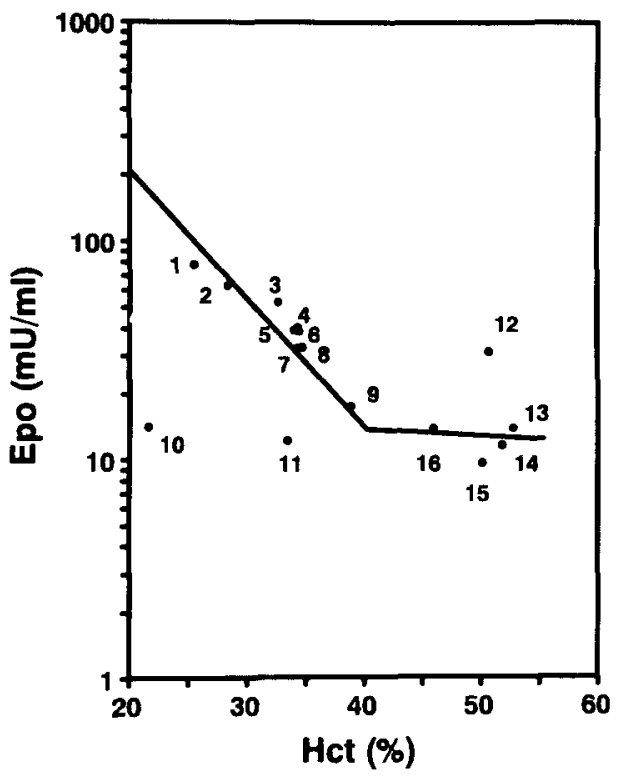

Fig 1. Relationship between EPO levels and Het in groups of patients, each represented by its mean values. The regression line displays the relationship in reference subjects (see text). 1, $\beta$-thalassemia/HbE; 2, AMM; 3, AIHA; 4, spherocytosis; 5, hypoplastic anemia; 6, MDS; 7, HbH disease; 8, BMT; 9, MPD; 10, renal failure; 11 , renal failure on rHuEpo; 12 , secondary $P C: 13$, relative $P C ; 14$, PC of uncertain origin; 15, PV; 16, normal subjects.

(ANOVA), with Snedecor's F-test or Welch's test as appropriate, was used to compare more than two groups. The $r$ correlation coefficients between Hct and $\log$ (Epo), $\log$ (TfR), and $\log$ (ETU) were computed in least squares regression equations. The slopes of the regression lines were tested for equality among groups by Student's $t$-tests in an analysis of covariance.

\section{RESULTS}

\section{Construction of Reference Regressions}

To construct the reference curves representing the relationships between Hct on the one hand, and Epo, TfR, and ETU on the other, only a subset of the subjects were considered. Patients with polycythemia were excluded, because abnormal marrow proliferation and excessive Epo production are often part of the pathophysiology of the disease. Patients with renal failure or hypoplastic anemia (as well as BMT patients) were excluded because of likely impairment of Epo production or reduced erythroid activity (see clinical criteria below). Patients with AMM, MDS, or advanced CML were also excluded because of the possibility of a component of relative erythroid hypoproliferation (see definition below) in many of them. Therefore, only the groups of normal subjects and of patients with other MPDs, AIHA, spherocytosis, $\beta$-thalassemia, and $\mathrm{HbH}$ disease were included. Among these groups, 3 patients were later excluded because Epo (in 2 $\beta$-thalassemic patients) or TfR (in 1 AIHA patient) were well beyond $3 \mathrm{SD}$ below the predicted value.

As shown in Fig 1, two least squares regression equations between Epo and Hct were computed, one for Hct less than
$40 \%$ and the other for Hct greater than $40 \%$. This cutoff Hct was chosen because it allowed for the best correlation for Epo data and because of literature data indicating that beyond such a Hct there is little modification of Epo levels. ${ }^{2,17}$ For Hct less than $40 \%$, the following regression $(r=.83, P=$ $.0000)$ was obtained between Epo $(\mathrm{mU} / \mathrm{mL})$ and Hct $(\%)$ : $\log ($ Epo $)=3.420-(0.056 \mathrm{Hct})$. For Hct greater than $40 \%$, the regression equation $(r=.12, \mathrm{NS})$ was: $\log ($ Epo $)=1.311$ $-(0.003 \mathrm{Hct})$. The reference regressions for TfR (Fig 2$)$ and ETU were calculated in the same subjects, using the same cutpoint Hct. The following regressions were obtained with $\operatorname{TfR}(\mu \mathrm{g} / \mathrm{L}): \log (\mathrm{TfR})=6.146-(0.057 \mathrm{Hct})$, when Hct is less than $40 \%(r=.75, P=.0000)$; and $\log (\mathrm{TfR})=4.079-$ $(0.008 \mathrm{Hct})$, when Hct is greater than $40 \%(r=.18$, NS). The following regressions were obtained with $\mathrm{ETU}(\mu \mathrm{mol} / \mathrm{L} / \mathrm{d})$ : $\log (\mathrm{ETU})=4.061-(0.052 \mathrm{Hct})$, when Hct is less than $40 \%$ $(r=-.64, P=.0000)$; and $\log (\mathrm{ETU})=2.086-(0.006 \mathrm{Hct})$, when Hct is greater than $40 \%(r=-.15$, NS). Thus the regression obtained for TfR and ETU had slopes very similar to those obtained with Epo ( $P>.1$ for differences in slope). Based on these formulas, predicted $\log (\mathrm{Epo}), \log (\mathrm{TfR})$, and $\log (E T U)$ values were derived for each sample and $\mathrm{O} / \mathrm{P}$ ratios of $\log$ (Epo), $\log$ (TfR), or $\log$ (ETU) were calculated. Ninetyfive percent confidence limits were calculated to define a range of reference values for individual $\mathrm{O} / \mathrm{P}$ values. These limits were 0.80 to 1.22 for $\mathrm{O} / \mathrm{P}$ Epo, 0.91 to 1.09 for $\mathrm{O} / \mathrm{P} \mathrm{TfR}$, and 0.76 to 1.22 for O/P ETU.

\section{Definition of Patterns of Erythropoiesis}

The major patterns of erythropoiesis were defined experimentally, based on the Hct, O/P TfR, and O/P Epo. The

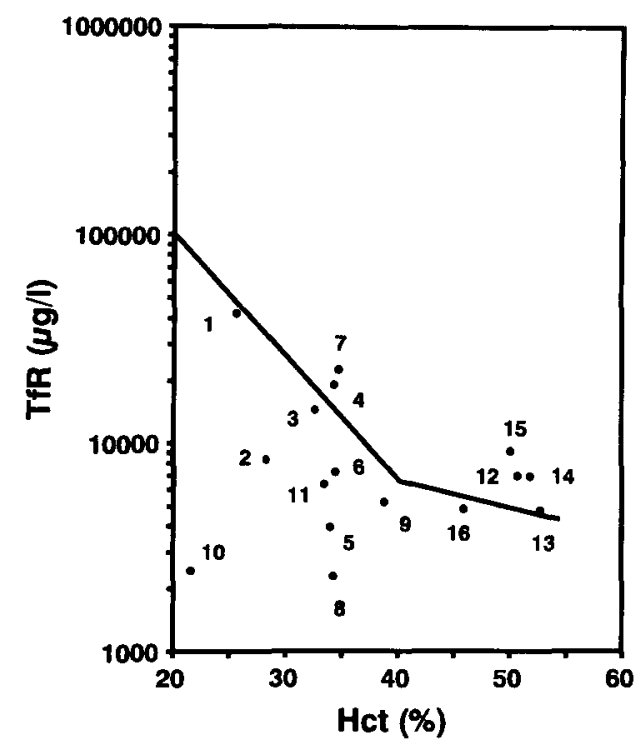

Fig 2. Relationship between TfR levels and Hct in groups of patients, each represented by its mean values. The regression line displays the relationship in reference subjects (see text). $1, \beta$-thalassemia/HbE; 2, AMM; 3, AlHA; 4, spherocytosis; 5, hypoplastic anemia; 6, MDS; 7, HbH disease; 8, BMT; 9, MPD; 10, renal failure; 11 , renal failure on rHuEpo; 12 , secondary $P C ; 13$, relative PC; 14 , PC of uncertain origin; 15, PV: 16, normal subjects. 
Table 1. Hematologic Values (mean \pm SD) in Groups of Subjects

\begin{tabular}{lrclccccc}
\hline & $N$ & $H c t(\%)$ & Epo $(\mathrm{mU} / \mathrm{mL})$ & TfR $(\mu \mathrm{g} / \mathrm{L})$ & ETU $(\mu \mathrm{mol} / \mathrm{L} / \mathrm{d})$ & $\mathrm{S}(\%)$ & Ferritin $(\mu \mathrm{g} / \mathrm{L})$ & Retic $(\%)$ \\
\hline Normal subjects & 31 & $45.9 \pm 3.6$ & $14.1 \pm 2.8$ & $4,970 \pm 1,030$ & $61 \pm 15$ & $30 \pm 8$ & $95 \pm 74$ & $2.1 \pm 1.2$ \\
Uncertain PC & 12 & $51.8 \pm 5.9$ & $12.1 \pm 4.2$ & $7,280 \pm 2,480$ & $77 \pm 20$ & $32 \pm 15$ & $92 \pm 81$ & $2.0 \pm 1.0$ \\
PV & 9 & $50.1 \pm 7.8$ & $10.1 \pm 3.6$ & $10,480 \pm 5,870$ & $97 \pm 31$ & $18 \pm 9$ & $40 \pm 17$ & $2.6 \pm 1.6$ \\
Secondary PC & 12 & $50.7 \pm 4.2$ & $52.9 \pm 77.9$ & $7,780 \pm 3,910$ & $80 \pm 30$ & $32 \pm 18$ & $162 \pm 196$ & $2.6 \pm 3.0$ \\
Relative PC & 5 & $52.7 \pm 2.1$ & $14.2 \pm 3.9$ & $4,840 \pm 890$ & $81 \pm 21$ & $35 \pm 8$ & $135 \pm 103$ & $1.8 \pm 1.6$ \\
MPD & 18 & $38.2 \pm 3.6$ & $21.6 \pm 20.3$ & $5,370 \pm 1,140$ & $76 \pm 65$ & $30 \pm 19$ & $510 \pm 1,070$ & $2.0 \pm 1.3$ \\
MDS & 10 & $34.6 \pm 4.7$ & $38.0 \pm 20.3$ & $7,980 \pm 6,750$ & $98 \pm 105$ & $47 \pm 26$ & $446 \pm 708$ & $3.3 \pm 3.8$ \\
AMM & 18 & $29.6 \pm 5.0$ & $74.5 \pm 87.2$ & $\mathbf{8 , 0 5 0} \pm 2,270$ & $133 \pm 64$ & $35 \pm 21$ & $276 \pm 280$ & $3.9 \pm 2.6$ \\
Hypoplastic anemia & 11 & $33.9 \pm 6.1$ & $88.9 \pm 78.7$ & $4,010 \pm 1,410$ & $50 \pm 18$ & $60 \pm 23$ & $550 \pm 530$ & $2.5 \pm 2.4$ \\
Renal failure & 15 & $21.6 \pm 3.4$ & $15.0 \pm 5.0$ & $2,610 \pm 1,060$ & $40 \pm 28$ & $36 \pm 19$ & $699 \pm 770$ & $2.1 \pm 1.0$ \\
Renal failure on rHuEpo & 5 & $33.4 \pm 3.6$ & $12.7 \pm 4.1$ & $6,410 \pm 860$ & $71 \pm 20$ & $23 \pm 6$ & $189 \pm 217$ & $2.5 \pm 1.0$ \\
BMT & 9 & $34.3 \pm 4.8$ & $51.8 \pm 69.5$ & $2,450 \pm 1,000$ & $63 \pm 36$ & $58 \pm 23$ & $2,925 \pm 2,399$ & $3.4 \pm 1.5$ \\
$\beta$-Thalassemia/HbE & 11 & $24.5 \pm 5.0$ & $80.4 \pm 39.7$ & $46,830 \pm 11,730$ & $562 \pm 207$ & $80 \pm 26$ & $1,686 \pm 1,954$ & $1.7 \pm 1.3$ \\
HbH disease & 10 & $33.0 \pm 2.7$ & $34.9 \pm 15.1$ & $23,250 \pm 5,760$ & $235 \pm 106$ & $57 \pm 24$ & $656 \pm 809$ & $2.0 \pm 1.7$ \\
Spherocytosis & 8 & $34.3 \pm 7.0$ & $44.6 \pm 20.4$ & $22,050 \pm 11,040$ & $506 \pm 303$ & $44 \pm 11$ & $923 \pm 698$ & $4.7 \pm 1.5$ \\
AlHA & 11 & $32.6 \pm 7.0$ & $97.5 \pm 147.1$ & $16,300 \pm 7,270$ & $235 \pm 133$ & $33 \pm 13$ & $535 \pm 635$ & $7.1 \pm 3.4$ \\
\hline
\end{tabular}

retic index and $T f R$ served to separate major patterns into variants. A normal pattern of erythropoiesis (pattern A) was defined by normal Hct, $\mathrm{O} / \mathrm{P}$ Epo, and $\mathrm{O} / \mathrm{P}$ TfR. The TfR value helped differentiate a variant of compensated hemolysis (pattern A2) in which TfR was increased, with a corresponding clinical picture of increased erythroid marrow cellularity and decreased RBC life span.

The term "hyperdestruction" referred to an experimental diagnosis (pattern $B$ ) in which Hct was decreased and $\mathrm{O} / \mathrm{P}$ Epo and O/P TfR were normal. The corresponding clinical diagnosis of increased RBC destruction and erythroid activity was based on the following criteria: (1) increased erythroid cellularity on marrow examination; (2) shortened RBC life span for a diagnosis of hemolysis. The retic index served to break this group down into two variants of "hemolysis" (pattern B1) and "ineffective erythropoiesis" (pattern B3). When the retic index was between 2 and 3, this distinction could not be readily made (pattern $\mathrm{B} 2$ ).

The term "intrinsic marrow hypoproliferation" referred to an experimental diagnosis (pattern $\mathrm{C}$ ) defined by erythropoietic activity inadequately low for the degree of anemia (decreased O/P TfR) in the presence of adequate Epo stimulation. Intrinsic marrow hypoproliferation could be absolute (decreased TfR), relative (increased $T f R$ ), or intermediate (normal TfR). In the first case, the experimental diagnosis was "hypoproliferative anemia" (pattern C3), with a corresponding clinical picture of reduced erythroid cellularity on marrow examination. In the second case, the experimental diagnosis was "mixed disorder of erythropoiesis" (pattern $\mathrm{C1}$ ), ie, with both hyperdestruction and hypoproliferation components. Clinically, the erythroid marrow could be normal or hypercellular. In the third case (pattern C2), the methodology could not distinguish between "mixed disorder of erythropoiesis" and "hypoproliferative anemia"; such patients all showed a retic index of less than 3.

The term "defective Epo production" referred to an experimental diagnosis (pattern $D$ ) defined by erythropoietic activity inadequately low for the degree of anemia (decreased
$\mathrm{O} / \mathrm{P}$ TfR) because of inadequate Epo production (decreased O/P Epo). Defective Epo production could be simple (decreased TfR), composite (increased TfR), or intermediate (normal TfR). As with pattern $\mathrm{C}$, the experimental diagnoses were Epo-dependent "hypoproliferative anemia" (pattern D3), "mixed disorder of erythropoiesis" (pattern D1), and indeterminate (pattern D2). The corresponding erythroid marrow should be respectively: hypocellular; normocellular or hypercellular; and normocellular or hypocellular. The retic index was always less than 3 in this group.

\section{"Experimental" Classification of Anemic Subjects}

Analysis of groups of subjects. Hematologic measurements are presented in Table 1. Epo, TfR, and ETU levels of each group were compared with values predicted from their Hct according to the reference regression equations defined above, and ratios of O/P Epo, TfR, ETU, and RCM are shown in Table 2. The figures illustrate the relationship between mean Hct and mean Epo (Fig 1) or mean TfR (Fig 2) levels for the various groups of subjects, as compared with the predicted values.

The 31 normal subjects had a mean (mean \pm SD) Hct of $45.9 \% \pm 3.6 \%$ (range, $40 \%$ to $52 \%$ in men and $37 \%$ to $47 \%$ in women), a mean Epo of $14.1 \pm 2.8 \mathrm{mU} / \mathrm{mL}$ (range, 9.3 to 23.1), a mean TfR of $4,970 \pm 1,030 \mu \mathrm{g} / \mathrm{L}$ (range, 2,700 to 7,650 ), and a mean ETU of $61 \pm 15 \mu \mathrm{mol} / \mathrm{L} / \mathrm{d}$ (range, 32 to 95). Their mean $\mathrm{O} / \mathrm{P}$ Epo was $0.99 \pm 0.07$ (range, 0.85 to 1.17), their mean $\mathrm{O} / \mathrm{P}$ TfR was $0.99 \pm 0.03$ (range, 0.91 to 1.05), and their mean $\mathrm{O} / \mathrm{P}$ ETU was $0.98 \pm 0.08$ (range, 0.86 to 1.13 ).

Patients with renal failure had Epo levels normal in absolute value (Table 1), but inadequate for the degree of anemia, as illustrated in Table 2 (decreased O/P Epo) and Fig 1 (mean value well below the reference line). Those treated with recombinant Epo came closer to the line of prediction (Fig 1). All other groups of anemic subjects had increased Epo values as compared with normal subjects (Table 1). This increase was appropriate for the degree of anemia, as shown 
From www.bloodjournal.org by Yves Beguin on February 4, 2009. For personal use only.

Table 2. Ratios of Observed to Predicted Values (mean \pm SD) in Groups of Subjects

\begin{tabular}{lrcccc}
\hline & $\mathrm{N}$ & O/P RCM & O/P Epo & O/P TfR & O/P ETU \\
\hline Normal subjects & 31 & $1.03 \pm 0.08$ & $0.99 \pm 0.07$ & $0.99 \pm 0.03$ & $0.98 \pm 0.08$ \\
Uncertain PC & 12 & $1.34 \pm 0.20^{*}$ & $0.93 \pm 0.12$ & $1.05 \pm 0.05 \dagger$ & $1.06 \pm 0.09^{*}$ \\
PV & 9 & $1.36 \pm 0.28 \ddagger$ & $0.85 \pm 0.11^{*}$ & $1.08 \pm 0.09^{*}$ & $1.10 \pm 0.11^{*}$ \\
Secondary PC & 12 & $1.37 \pm 0.33^{*}$ & $1.31 \pm 0.37 \ddagger$ & $1.05 \pm 0.06^{*}$ & $1.07 \pm 0.08^{*}$ \\
Relative PC & 5 & $1.17 \pm 0.09^{*}$ & $1.01 \pm 0.12$ & $1.01 \pm 0.02$ & $1^{*} .09 \pm 0.07^{*}$ \\
MPD & 18 & $0.92 \pm 0.17 \ddagger$ & $1.00 \pm 0.19$ & $0.94 \pm 0.05 \dagger$ & $0.86 \pm 0.16^{*}$ \\
MDS & 10 & $0.79 \pm 0.14 \dagger$ & $1.00 \pm 0.15$ & $0.92 \pm 0.04 \dagger$ & $0.80 \pm 0.14 \dagger$ \\
AMM & 18 & $0.85 \pm 0.23 \ddagger$ & $0.93 \pm 0.23$ & $0.88 \pm 0.05 \dagger$ & $0.83 \pm 0.15 \dagger$ \\
Hypoplastic anemia & 11 & $0.75 \pm 0.17 \dagger$ & $1.02 \pm 0.29$ & $0.85 \pm 0.06 \dagger$ & $0.75 \pm 0.14 \dagger$ \\
Renal failure & 15 & $0.53 \pm 0.09 \dagger$ & $0.53 \pm 0.10 \dagger$ & $0.69 \pm 0.04 \dagger$ & $0.51 \pm 0.15 \dagger$ \\
Renal failure on rHuEpo & 5 & $0.80 \pm 0.10 \dagger$ & $0.72 \pm 0.16 \dagger$ & $0.90 \pm 0.06^{*}$ & $0.80 \pm 0.12 \dagger$ \\
BMT & 9 & $0.64 \pm 0.13 \dagger$ & $1.01 \pm 0.21$ & $0.81 \pm 0.06 \dagger$ & $0.78 \pm 0.12 \dagger$ \\
$\beta-$ Thalassemia/HbE & 11 & $\mathrm{NA}$ & $0.94 \pm 0.13$ & $0.98 \pm 0.06$ & $0.97 \pm 0.06$ \\
HbH disease & 10 & $\mathrm{NA}$ & $1.03 \pm 0.11$ & $1.05 \pm 0.04^{*}$ & $1.04 \pm 0.09$ \\
Spherocytosis & 8 & $0.91 \pm 0.08$ & $1.04 \pm 0.12$ & $1.01 \pm 0.04$ & $1.10 \pm 0.08^{*}$ \\
AlHA & 11 & $0.84 \pm 0.15^{*}$ & $1.05 \pm 0.10$ & $0.98 \pm 0.09$ & $1.01 \pm 0.14$ \\
\hline
\end{tabular}

$P$ values are given for comparison with normal subjects.

Abbreviation: $\mathrm{NA}$, not available.

$* P<.01$.

$\dagger P<.001$

$\ddagger P<.05$.

in Fig 1 (all groups were near the line of prediction) and in Table 2 (O/P Epo close to 1.0).

Patients with AIHA, spherocytosis, $\beta$-thalassemia, and $\mathrm{HbH}$ disease had TfR and ETU values considerably elevated as compared with normal subjects (Table 1), indicating an increased erythroid marrow activity in response to an increased Epo stimulation. The distinction between hemolysis and ineffective erythropoiesis could be made by a retic index of greater than 3 and less than 2, respectively.

On the other hand, only the BMT, hypoplastic anemia, and renal failure groups had decreased TfR values (Table 1). They were also the ones that fell the farthest below the reference line (Fig 2) and had the lowest O/P TfR and O/P ETU (Table 2). Therefore, the anemia in these groups appeared to be a consequence of decreased erythroid activity, either because of reduced Epo production (renal failure) or intrinsic erythroid marrow hypoproliferation (BMT and hypoplastic anemia).

Patients with MDS or AMM had elevated TfR levels (Table 1), demonstrating that their anemia was not primarily due to reduced erythroid activity. However, their level of erythropoiesis remained insufficient for the degree of anemia (Table
2 and Fig 2). This indicated that, despite adequate Epo stimulation, there was a hypoproliferative component to their anemia. Hence, the pathophysiology of their anemia was mixed, ie, a combination of hyperdestruction and hypoproliferation.

Analysis of individual patients. Individual patients were classified according to their experimental pattern of erythropoiesis. Table 3 discloses typical cases illustrating the various patterns, whereas Table 4 dissects out each clinical group into experimental diagnostic groups. All normal subjects had a normal pattern of erythropoiesis (pattern A1). Of 11 patients with AIHA, 7 had typical hemolysis (pattern B1), 1 had an intermediate retic index (pattern B2), 2 had the variant normal pattern with increased TfR (pattern A2), indicating that hemolysis was completely compensated by increased erythroid activity, and one had an important intrinsic marrow hypoproliferative component (pattern $\mathrm{C1}$ ), due to metastatic breast cancer. Patients with spherocytosis had typical hemolysis (pattern B1), except for one who had returned to normal erythropoiesis (pattern A1) after splenectomy. Patients with $\beta$-thalassemia or $\mathrm{HbH}$ disease had typical ineffective erythropoiesis (pattern $\mathrm{B} 3$ ). However, in one $\mathrm{HbH}$ pa-

Table 3. Some Typical Cases and Characteristic Exceptions to the Patterns of Erythropoiesis

\begin{tabular}{|c|c|c|c|c|c|c|}
\hline Pattern of Erythropoiesis & Clinicat Diagnosis & Hct & O/P TfR & O/P Epo & TfR & Retic Index \\
\hline Normal (A1) & Normal subject & 48.8 & 1.00 & 1.04 & 4,670 & 2.1 \\
\hline Hemolysis (B1) & AlHA & 31.1 & 1.00 & 0.94 & 24,000 & 4.1 \\
\hline Ineffective erythropoiesis (B3) & $\mathrm{HbH}$ disease & 30.0 & 0.99 & 0.91 & 24,650 & 0.9 \\
\hline Intrinsic marrow hypoproliferation: hypoproliferative anemia (C3) & Aplastic anemia & 25.2 & 0.70 & 1.04 & 1,980 & 0.7 \\
\hline Intrinsic marrow hypoproliferation: mixed disorder of erythropoiesis (C1) & AMM & 23.0 & 0.84 & 1.03 & 11,840 & 1.5 \\
\hline Defective Epo production: hypoproliferative anemia (D3) & Renal failure & 20.6 & 0.68 & 0.51 & 2,500 & 1.0 \\
\hline Defective Epo production: mixed disorder of erythropoiesis (D1) & $\beta$-Thalassemia + infection & 22.0 & 0.89 & 0.74 & 22,600 & 0.8 \\
\hline
\end{tabular}


Table 4. Number of Individual Patients by Clinical Diagnosis in Each Pattern of Erythropoiesis

\begin{tabular}{|c|c|c|c|c|c|c|c|c|c|c|c|c|}
\hline \multirow[b]{2}{*}{ Variant } & & \multicolumn{2}{|c|}{$\begin{array}{c}\text { A } \\
\text { Normal }\end{array}$} & \multicolumn{3}{|c|}{$\begin{array}{c}\text { B } \\
\text { Hyperdestruction }\end{array}$} & \multicolumn{3}{|c|}{$\begin{array}{c}\text { C } \\
\text { Intrinsic Marrow } \\
\text { Hypoproliferation }\end{array}$} & \multicolumn{3}{|c|}{$\begin{array}{c}\text { D } \\
\text { Defective Epo } \\
\text { Production }\end{array}$} \\
\hline & & 1 & 2 & 1 & 2 & 3 & 1 & 2 & 3 & 1 & 2 & 3 \\
\hline $\mathrm{Hct}$ & & \multicolumn{2}{|c|}{$\mathrm{N}$} & \multicolumn{3}{|c|}{ Low } & \multicolumn{3}{|c|}{ Low } & \multicolumn{3}{|c|}{ Low } \\
\hline O/P TfR & & \multicolumn{2}{|c|}{$\mathrm{N}$-(High) } & \multirow{2}{*}{\multicolumn{3}{|c|}{$\mathrm{N}$}} & \multicolumn{3}{|c|}{ Low } & \multicolumn{3}{|c|}{ Low } \\
\hline O/P Epo & & \multicolumn{2}{|c|}{$\mathrm{N}$-(High) } & \multicolumn{2}{|r|}{$\mathrm{N}$} & & \multicolumn{3}{|c|}{$\mathrm{N}$} & \multicolumn{3}{|c|}{ Low } \\
\hline TfR & & $\mathrm{N}$ & High & & & & High & $\mathrm{N}$ & Low & High & $\mathrm{N}$ & Low \\
\hline Retic index & & & & $>3$ & $2-3$ & $<2$ & & & & & & \\
\hline Erythroid cellularity & & & High & High & High & High & High-N & N-Low & Low & High-N & N-Low & Low \\
\hline Mean RBC life span & & & Low & Low & & & & & & & & \\
\hline Normal subjects & 31 & 31 & & & & & & & & & & \\
\hline MPD & 18 & 7 & & & 1 & 5 & & 4 & & & 1 & \\
\hline MDS & 10 & 1 & & 1 & & 4 & 1 & 3 & & & & \\
\hline AMM & 18 & 1 & & 1 & 1 & & 7 & 5 & & 1 & 2 & \\
\hline Hypoplastic anemia & 11 & 2 & & & & & & 6 & 1 & & & 2 \\
\hline Renal failure & 15 & & & & & & & & & & 4 & 11 \\
\hline Renal failure on rHuEpo & 5 & 1 & & & 1 & & & & & & 3 & \\
\hline BMT & 9 & $1^{*}$ & & & & & & 1 & 6 & & 1 & \\
\hline$\beta$-Thalassemia/HbE & 11 & & & & & 9 & & & & 2 & & \\
\hline $\mathrm{HbH}$ disease & 10 & & & 1 & & 9 & & & & & & \\
\hline Spherocytosis & 8 & 1 & & 7 & & & & & & & & \\
\hline AlHA & 11 & & $2 \dagger$ & 7 & 1 & & 1 & & & & & \\
\hline
\end{tabular}

*High O/P Epo.

tHigh O/P TfR.

tient, a high retic index suggested predominantly peripheral hemolysis (pattern B1) instead, and in two $\beta$-thalassemia patients, a component of defective Epo stimulation (pattern D1) was identified. Renal failure was typically associated with Epo-dependent hypoproliferative anemia (pattern D3), whereas a minority of them managed to achieve normal (although insufficient) TfR levels (pattern D2). Treatment with recombinant Epo reversed this pattern completely in 1 patient (pattern A1). In another patient, exogenous Epo produced the apparently aberrant situation of normal erythropoiesis (O/P TfR 0.94) despite insufficient (endogenous) Epo stimulation (O/P Epo 0.65). BMT was mostly associated with hypoproliferative anemia of marrow origin (pattern C3), whereas 1 patient had an additional component of defective Epo production (pattern D2). One BMT patient had normalized erythropoiesis (pattern A 1) through an inappropriate increase of Epo production (O/P Epo 1.47) induced by lung disease. The majority of patients with hypoplastic anemia had hypoproliferative anemia, either by intrinsic marrow defect (patterns C2 and C3) or by defective Epo production (pattern D3) in a case of hypothyroidism or a case of anemia of chronic disorder. In addition, two patients in this group had completely normalized at the time of the study (pattern A1). Many patients with MPD had a normal pattern (pattern A1), but ineffective erythropoiesis (pattern B3) and a component of intrinsic marrow hypoproliferation (pattern C2) were often encountered. Ineffective erythropoiesis (pattern B3) was the predominant mechanism of anemia in MDS, but hemolysis (pattern B1) and intrinsic marrow hypopro- liferation (patterns $\mathrm{C} 1$ and $\mathrm{C} 2$ ) were also seen. In AMM, hyperdestruction (pattern B), intrinsic marrow hypoproliferation (pattern C), and defective Epo production (pattern D) were all observed as the primary mechanism of anemia, as well as in combination.

\section{"Experimental" Classification of Polycythemic Subjects}

Groups of polycythemia patients had elevated Hct (Table 1) and RCM (Table 2). Patients with a clinical diagnosis of relative $\mathrm{PC}$ had normal (Table 1) and appropriate (Figs 1 and 2 and Table 2) Epo and TfR levels. Patients with a clinical diagnosis of absolute PC had increased TfR and ETU (Table 1) in the presence of high $\mathrm{O} / \mathrm{P}$ Epo in the case of secondary $\mathrm{PC}$, or a clinical diagnosis of normal to low to normal $\mathrm{O} / \mathrm{P}$ Epo in the case of PV or uncertain PC (Fig 1 and Table 2). Clinical diagnosis of $\mathrm{PC}$ was also compared with experimental diagnosis on an individual basis. Inappropriately high Epo (increased O/P Epo) was considered characteristic of secondary $\mathrm{PC}$, whereas inappropriately high TfR (increased $\mathrm{O} / \mathrm{P}$ $\mathrm{TfR}$ ) in the presence of normal to low to normal O/P Epo was considered characteristic of PV. Eight patients had Hct and RCM within normal limits at time of investigation, and 6 had increased Hct but normal RCM because of previous therapy. O/P Epo and O/P TfR were normal in all of them. Among patients who were polycythemic at the time of investigation, there was no experimental confusion between PV and secondary PC. Among patients with uncertain PC, two were identified as having likely PV (increased O/P TfR with normal, to low to normal O/P Epo). 


\section{Correlations Between Parameters of Erythropoiesis}

There was an excellent correlation between Hct and O/P $\operatorname{RCM}(r=.83, P<.0001)$, as well as between ETU and TfR levels $(r=.82, P<.0001)$. Expressing ETU values in micromoles per day and TfR in micrograms, by multiplying concentrations by blood or plasma volumes, respectively, did not alter the correlation $(r=.81, P<.0001)$ between the two measurements of erythropoiesis. This is illustrated in Fig 3 , in which each group of subjects is represented by its mean ETU and TfR values. In polycythemia patients, there was a positive correlation between TfR and Hct $(r=.42, P<.05)$ as well as between TfR and RCM $(r=.63, P<.001)$. In contrast to other groups of patients, there was a positive relationship between Epo levels and Hct in patients with renal failure $(r=.46, P<.05)$. The overall correlations between Epo levels and TfR levels $(r=.45, P<.001)$ or ETU $(r=$ $.42, P<.001)$ were relatively weak because they reflected many different patterns of erythropoiesis.

\section{DISCUSSION}

In the present study, we investigated the pathophysiology of RBC production in a large group of normal, anemic, or polycythemic subjects. The RCM was quantitated by direct isotopic measurement, Epo stimulation by serum Epo levels, and total marrow erythropoietic activity by the ferrokinetic measurement of the ETU, serum TfR levels, and retic index. Instead of only quantitating Epo levels and erythropoiesis in absolute terms, we also evaluated them in relation to the degree of anemia or PC, and expressed results as a ratio of observed to predicted values. These quantitative measurements were supplemented by the qualitative evaluation of erythroid cellularity on marrow examination. The study of anemic subjects is based on a simple model of the regulation of erythropoiesis, ie, the RCM determines Epo production, which stimulates erythropoietic activity. If this model of erythropoiesis is valid from a quantitative point of view, then the relationship between Hct and erythropoiesis should be similar to the one between Hct and Epo. If this is the case, then (1) an inverse relationship should be observed between Epo and Hct, as well as between TfR and Hct; (2) the regression lines of $\log (\mathrm{Epo})$ versus Hct and of $\log (\mathrm{TfR})$ versus Hct should have similar slopes; and (3) Epo and TfR values predicted from the regressions should then not be different from observed values. These three conditions were indeed fulfilled. First, after excluding groups of patients whose anemia was known to be associated with renal failure and/or a hypoproliferative component, an excellent inverse correlation was observed between Hct and Epo on the one hand, and between Hct and TfR (as well as ETU) on the other. Second, the slope of the regression of TfR (as well as ETU) versus Hct was very similar to the slope of the regression of Epo versus Hct. Third, Epo and TfR (as well as ETU) values predicted from the regression equations were quite comparable to observed values in most groups of subjects (Table 2 and Figs 1 and 2). A predictable exception for Epo was the group of renal failure patients (O/P Epo $53 \%$ of predicted), whereas those treated with recombinant Epo came closer to the line of prediction (O/P Epo $72 \%$ of predicted) (Fig 1). For TfR, the groups of patients whose primary cause of anemia was decreased erythroid activity fell the farthest below prediction, whereas patients with MDS and AMM also had a hypoproliferative component to their anemia. All this is in keeping with our knowledge of the pathophysiology of these hematologic disorders.

When normal individuals are phlebotomized while iron supply is adequate, they are able to sustain an erythropoietic activity 3 to 5 times normal. ${ }^{18}$ This will translate into TfR levels increased in proportion to Epo stimulation. However, in the presence of erythroid hypoproliferation, TfR values will be lower than expected from the level of Epo stimulation. In the current study, only 20 patients had purely hypoproliferative anemia (decreased TfR), whereas all others had
Fig 3. Correlation between TfR levels and ETU in groups of patients, each represented by its mean values. The shaded area represents a proportional relationship, and its limits were obtained by drawing a line through mean \pm SD TfR levels and ETU values in normal subjects (open circle). 1, $\beta$-thalassemia/ HbE; 2, AMM; 3, AlHA; 4, spherocytosis; 5, hypoplastic anemia; 6, MDS; 7, HbH disease; 8, BMT; 9. MPD; 10, renal failure; 11 , renal failure on rHuEpo: 12, secondary PC; 13 , relative PC: $14, P C$ of uncertain origin; 15, PV. ETU is overestimated in BMT recipients because of hyperferremia.

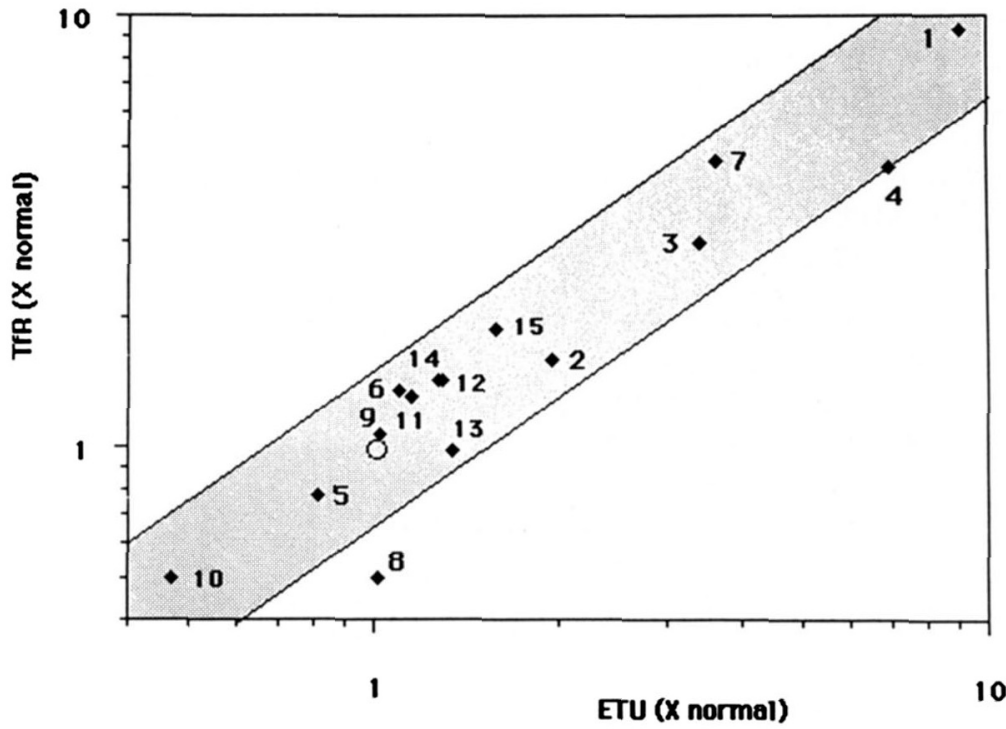


normal or elevated TfR values. However, when examined relative to the degree of anemia, 42 additional patients were found to have varying degrees of erythroid marrow hypoproliferation, including 16 patients who actually had elevated serum TfR levels. Therefore, any measurement of erythropoiesis not related to the degree of anemia could grossly overestimate marrow proliferative capacity. Several attempts have been made previously to address this problem. Using sophisticated computer analysis of ferrokinetic data, a distinction could be made between anemic patients with high and low potential erythropoiesis on the basis of a value for total erythropoiesis higher or lower than 4 times basal, and a more precise rule could be derived by defining a decision boundary relative to the hemoglobin concentration. ${ }^{19}$

Examining serum Epo and TfR in relation to the degree of anemia had the further advantage of identifying patients with a "mixed disorder of erythropoiesis," ie, individuals with a hypoproliferative component combined with a component of hyperdestruction (hemolysis or ineffective erythropoiesis). Thus, besides typical patterns of erythropoiesis (Table 3), many patients had a composite pathophysiology. As shown in ferrokinetic studies, ${ }^{19}$ dissecting out groups of patients to examine individual patterns of erythropoiesis (Table 4) showed that the mechanism of anemia may be similar among patients with different diseases, but can also vary much among patients with the same clinical disorder. For instance, among patients with a clinical diagnosis of hemolytic anemia (spherocytosis or AIHA), 1 became normal after splenectomy, 2 compensated completely for the hyperdestruction, and 1 had a component of hypoproliferation. Besides patients with renal failure, defective Epo production was also observed in many patients, including some with $\beta$-thalassemia/ $\mathrm{HbE},{ }^{20} \mathrm{AMM}$, MPD, and hypoplastic anemia or after BMT (Table 4).

Apparently aberrant patterns could also find an explanation. For instance, a patient with renal failure had normal erythropoietic activity despite inadequate endogenous Epo production because of administration of exogenous recombinant Epo, a BMT patient had normalized erythroid activity through excessive Epo stimulation induced by chronic hypoxia, and 2 patients had elevated TfR levels despite an otherwise normal pattern of erythropoiesis because they had fully compensated for a hemolytic process.

The identification of different pathophysiologic components could have important implications for the treatment of the anemia in individual patients with an otherwise welldefined clinical diagnosis. For instance, treatment with rHuEpo would probably be unsuccessful in the case of a purely hyperdestructive anemia, but would be more likely to be effective when a component of defective Epo production or a purely hypoproliferative anemia have been identified. In the case of diseases such as AMM, MDS, or MPD, in which the pathophysiology of the anemia is often composite, a careful analysis could separate patients into poor and good candidates for Epo therapy. This remains to be shown prospectively.

The proposed model of erythropoiesis has several limitations. First, the exactness of each measurement has its own limits; an apparent inadequacy of Epo production could in fact result from better tissue oxygenation with altered oxygen dissociation curves, iron deficiency could cause some elevation of TfR levels not related to erythropoiesis, ${ }^{21}$ and the retic count is hampered by imprecision and can be at best considered as a semiquantitative measurement. Second, while a mixed disorder of erythropoiesis can be readily identified in patients with low O/P TfR but increased TfR value, it is more difficult to distinguish a component of hyperdestruction from a situation of blunted marrow response to increased Epo stimulation without hyperdestruction when TfR level is normal. However, when we carefully analyzed patterns of erythropoiesis in relation to clinical diagnosis (Table 4), it became apparent that when TfR was normal and Epo production inadequate (decreased $\mathrm{O} / \mathrm{P}$ Epo), blunted marrow responsiveness was by far more likely, whereas a component of hyperdestruction was more likely when $\mathrm{O} / \mathrm{P}$ Epo was normal.

A third difficulty is encountered when one attempts to separate hemolysis from ineffective erythropoiesis, as both are characterized by a low Hct, elevated TfR, and normal $\mathrm{O} / \mathrm{P}$ TfR and $\mathrm{O} / \mathrm{P}$ Epo. After appropriate corrections are made ${ }^{16}$ the retic index can be of great help in most cases. While the RBC life span can be evaluated by isotopic methods, there is no gold standard with which to measure ineffective erythropoiesis. Sophisticated computer analyses of ferrokinetic data have attempted to quantitate ineffective erythropoiesis, but some aspects of iron exchange remained impossible to resolve in these calculations, particularly the separation of wastage iron of erythropoiesis from iron taken up by parenchymal cells. ${ }^{22,23}$ Some cases presenting conditions defined as dyserythropoietic can also show a component of peripheral hemolysis, while so-called hemolytic anemias can be characterized by varying degrees of ineffective erythropoiesis. ${ }^{24-26}$ Therefore, the erythropoietic pattern may depend on the stage of erythroid development at which destruction occurs and a clear distinction between ineffective erythropoiesis and peripheral hemolysis may not always be possible.

Finally, the model does not contribute much to the approach to patients with $\mathrm{PC}$. The RCM correlated positively with TfR much better than with the Hct, suggesting a causative and quantitatively valid link between the RCM and erythropoiesis as measured by the TfR level. However, a typical pattern of increased O/P TfR with (secondary PC) or without (PV) increased O/P Epo was not frequently observed. Therefore, a correct diagnosis of PC should be based on an RCM determination (and not a Hct) and other standard criteria. ${ }^{7}$ Serum Epo could be useful in identifying patients with secondary PC, although many of them have normal values, ${ }^{2,27-29}$ and serum TfR in making a diagnosis of PV in some patients with $\mathrm{PC}$ of uncertain origin, as illustrated in two of our patients.

Given these limitations of a model incorporating the Hct, serum Epo, serum TfR, and retic index, it could be possible to substitute other measurements. The isotopic measurement of the RCM gives a better idea of the total number of RBCs than the Hct, particularly in the case of splenomegaly or altered plasma volume. Although a direct evaluation of the 
$\mathrm{RCM}$ is indispensable in the diagnosis of $\mathrm{PC}$, the Hct remains sufficient for most practical purposes in a situation of anemia. While we do not have any equivalent tool to replace serum Epo, erythropoiesis could be approached by other methods than TfR, such as the marrow erythroid cellularity, retic index, and ETU.

The present model only deals with the pathophysiology of anemia and has no pretension to replace a marrow examination to identify specific clinical disorders or to recognize iron deficiency along with a serum ferritin level. A marrow sample can also be used to evaluate overall erythroid cellularity, but can only describe it in a semiquantitative fashion as normal, hypocellular, or hypercellular. The erythropoietic response to anemia cannot be measured quantitatively from the marrow alone, and a hypoproliferative component cannot be identified in patients with normocellular or hypercellular erythroid marrow. Furthermore, there are many instances in which a representative sample cannot be obtained because of nonhomogeneous marrow distribution, or in which an abnormally expanded white blood cell population precludes a meaningful interpretation of the erythroid/myeloid ratio and the erythroid mass.

As illustrated in Table 1, the retic percentage does not provide a quantitative evaluation of total erythropoiesis, as the majority of the groups of patients were indistinguishable from normal individuals and several groups of patients characterized by hypoproliferative anemia as defined here had elevated counts. The retic count showed no correlation with the Hct, TfR, or ETU. After corrections were applied, a retic index greater than 3 was rarely encountered outside the AIHA and spherocytosis groups, although many of the patients had increased erythropoietic activity. Patients with a hypoproliferative component, ineffective erythropoiesis, or both remained indistinguishable. Therefore, with the exception of patients with a purely hemolytic anemia, the retic count and index alone do not provide a quantitative evaluation of erythropoiesis. However, when used in conjunction with quantitative measurements, they provide information complementary to $\mathrm{TfR}$, particularly when one desires to separate hemolysis from ineffective erythropoiesis.

When transferrin saturation decreases below $16 \%$ or the plasma radioiron $t-1 / 2$ is less than 30 minutes, ferrokinetic measurements are no longer valid. ${ }^{30}$ On the other hand, when plasma iron is elevated, the presence of nontransferrin-bound iron $^{31,32}$ whose turnover is not related to the erythron but to the hepatocyte ${ }^{33}$ can overestimate erythropoiesis. This problem was probably encountered in our BMT patients (Fig 3). Because its calculation is based on three separate measurements (plasma iron, radioiron $\mathrm{t}-1 / 2$, and $\mathrm{Hct}$ ), the ETU is more vulnerable to experimental error than TfR levels and, indeed, in the present study its coefficient of variation was higher in normal subjects as well as in patients, its correlation with Het weaker, and its $95 \%$ confidence limits wider. Although the overall correlation between ETU and TfR was excellent, the greater precision and simpler methodology of the TfR determination should make it the method of choice for estimating erythroid activity.

\section{ACKNOWLEDGMENT}

The authors thank Georges Weber (Department of Experimental Nuclear Physics, University of Liège) for preparing the figures.

\section{REFERENCES}

1. Eckardt K-U, Bauer C: Erythropoietin in health and disease. Eur J Clin Invest 19:117, 1989

2. Garcia JF, Ebbe SN, Hollander L, Cutting HO, Miller MO, Cronkite EP: Radioimmunoassay of erythropoietin: Circulating levels in normal and polycythemic human beings. J Lab Clin Med 99:624, 1982

3. Cazzola M, Pootrakul P, Huebers HA, Eng M, Eschbach J, Finch CA: Erythroid marrow function in anemic patients. Blood 69: 296, 1987

4. Beguin Y, Huebers HA, Josephson B, Finch CA: Transferrin receptors in rat plasma. Proc Natl Acad Sci USA 85:637, 1988

5. Huebers HA, Beguin Y, Pootrakul P, Einspahr D, Finch CA: Intact transferrin receptors in human plasma and their relation to erythropoiesis. Blood 75:102, 1990

6. Hurley PJ: Red cell and plasma volume in normal adults. J Nucl Med 16:46, 1975

7. Berlin NI: Diagnosis and classification of the polycythemias. Semin Hematol 12:339, 1975

8. Lipschitz DA, Cook JD, Finch CA: A clinical evaluation of serum ferritin as an index of iron stores. N Engl J Med 290:1213, 1974

9. ICSH: Recommended methods for measurement of red-cell and plasma volume. J Nucl Med 21:793, 1980

10. Bothwell TH, Conrad ME, Cook JD, Fielding J, Hallberg L, Izak G, Layrisse M, Ramsay WNM: Recommendations for measurement of serum iron in human blood. Br J Haematol 38:291, 1978

11. Bothwell TH, Conrad ME, Cook JD, Fielding J, Hallberg L, Izak G, Layrisse M, Ramsay WNM: The measurement of total and unsaturated iron-binding capacity in serum. $\mathrm{Br} \mathrm{J}$ Haematol 38:281, 1978

12. Cook JD, Marsaglia G, Eschbach JW, Funk DD, Finch CA: Ferrokinetics: A biologic model for plasma iron exchange in man. $\mathrm{J}$ Clin Invest 49:197, 1970

13. Cazzola M, Huebers HA, Sayers MH, MacPhail AP, Eng M, Finch CA: Transferrin saturation, plasma iron turnover, and transferrin uptake in normal humans. Blood 66:935, 1985

14. Huebers HA, Huebers E, Josephson B, Csiba E: A highly efficient chemical isolation procedure for the rat placental transferrin receptor. Biochim Biophys Acta 991:30, 1989

15. Axelsen NH, Kroll J, Weeke B: A manual of quantitative immunoelectrophoresis. Methods and applications. Scand J Immunol Suppl 2:1, 1973

16. Hillman RS, Finch CA: Red Cell Manual. Philadelphia, PA, F.A. Davis Co, 1985, p I

17. Nielsen OJ: Determination of human erythropoietin by radioimmunoassay. Methods and clinical data. Clin Chim Acta 176: 303, 1988

18. Hillman RS, Henderson PA: Control of marrow production by the level of iron supply. J Clin Invest 48:454, 1969

19. Barosi G, Cazzola M, Berzunini C, Quaglini S, Stefanelli M: Classification of anaemia on the basis of ferrokinetic parameters. $\mathrm{Br}$ J Haematol 61:357, 1985

20. Manor D, Fibach E, Goldfarb A, Rachmilewitz EA: Erythropoietin activity in the serum of beta thalassemic patients. Scand $J$ Haematol 37:221, 1986 
21. Flowers CH, Skikne BS, Covell AM, Cook JD: The clinical measurement of serum transferrin receptor. J Lab Clin Med 114: 368,1989

22. Orr JS, Horton PW, Hutcheon AW, Dagg JH: Erythropoiesis and iron kinetics. Br J Haematol 42:155, 1979

23. Beguin Y, Stray SM, Cazzola M, Huebers HA, Finch CA: Ferrokinetic measurement of erythropoiesis. Acta Haematol (Basel) 79:121, 1988

24. Stefanelli M, Barosi G, Cazzola M, Orlandi E: Quantitative assessment of erythropoiesis in haemolytic disease. $\mathrm{Br} \mathrm{J}$ Haematol 45:297, 1980

25. Cazzola M, Barosi G, Berzuini C, Dacco M, Orlandi E, Stefanelli M, Ascari E: Quantitative evaluation of erythropoietic activity in dysmyelopoietic syndromes. Br J Haematol 50:55, 1982

26. Barosi G, Cazzola M, Frassoni F, Orlandi E, Stefanelli M: Erythropoiesis in myelofibrosis with myeloid metaplasia: Recognition of different classes of patients by erythrokinetics. Br J Haematol 48 : 263,1981

27. Cotes PM, Doré CJ, Liu Yin JA, Lewis SM, Messinezy M, Pearson TC, Reid C: Determination of serum immunoreactive erythropoietin in the investigation of erythrocytosis. N Engl J Med $315: 283,1986$

28. Erslev AJ, Caro J: Pure erythrocytosis classified according to erythropoietin titers. Am J Med 76:57, 1984

29. Koeffler HP, Goldwasser E: Erythropoietin radioimmunoassay in evaluating patients with polycythemia. Ann Intern Med 94:44, 1981

30. Finch CA, Deubelbeiss K, Cook JD, Eschbach JW, Harker LA, Funk DD, Marsaglia G, Hillman RS, Slichter S, Adamson JW, Ganzoni A, Giblett ER: Ferrokinetics in man. Medicine 49:17, 1970

31. Hershko C, Graham G, Bates GW, Rachmilewitz EA: Nonspecific serum iron in thalassaemia: An abnormal serum iron fraction of potential toxicity. Br J Haematol 40:255, 1978

32. Pootrakul P, Josephson B, Huebers HA, Finch CA: Quantitation of ferritin iron in plasma, an explanation for non-transferrin iron. Blood 71:1120, 1988

33. Wright TL, Fitz JG, Weisiger RA: Non-transferrin-bound iron uptake by rat liver. Role of membrane potential difference. J Biol Chem 263:1842, 1988 